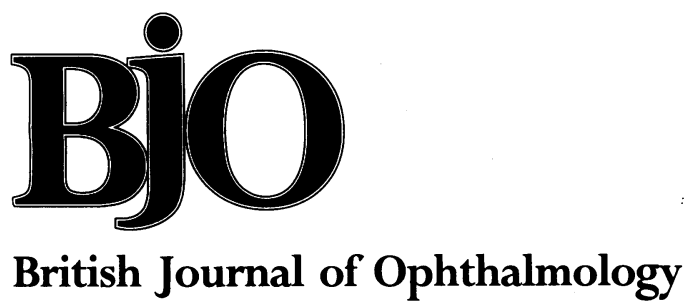

Editorials

\title{
What would Socrates have made of normal tension glaucoma?
}

The paper in this issue of the $B F O$ by Hitchings et al (p 402) on surgery for normal tension glaucoma is a valuable pragmatic study which shows the benefit of trabeculectomy. It should, however, lead us to contemplate the fundamental nature of the conditions we label low (or normal) tension glaucoma. Socrates might well have enjoyed a dialogue on this subject. He encouraged the idea that a concept only became valid once it could be defined in abstract terms rather than by reference to specific concrete examples. We are still far from that ideal in low tension glaucoma. Indeed, it is often the absence of certain features which guides our definition. This is hardly satisfactory as it may cause inadvertent inclusion of other diseases even in the more rigorous scientific studies.

True high pressure primary open angle glaucoma is itself difficult to define, especially in the early stages. ${ }^{1}$ The intraocular pressure fluctuates and a single sample measurement will often yield a normal result. ${ }^{2}$ The optic disc and visual field abnormalities depend on subjective methods of assessment with many causes for misinterpretation. Whether we should use the term tension or pressure is open to semantic argument.

Examination of the use of the word 'normal' rather than 'low' in this paper is more constructive. The first negative feature in the definition of this disease is the persistent absence of raised intraocular pressure yet Hitchings et al include patients with pressures up to $24 \mathrm{~mm} \mathrm{Hg}$ when examined by diurnal phasing. It is, therefore, likely that some of these cases are in the lower pressure ranges of true 'high pressure' primary open angle glaucoma. Despite the poor quality of many treatment trials, ${ }^{3}$ this disease is known to benefit from medical or surgical treatment to lower the pressure. ${ }^{4}$ The present study, although of considerable size for such a thorough investigation of this rare disease, is too small to allow separation of the results in unequivocal low pressure eyes from those who have pressures which are sometimes slightly raised.

The authors have followed the sequence of investigation necessary in low tension glaucoma. In addition to a continued search for raised pressure it is recommended ${ }^{5}$ that several features must be excluded: a history of eye disease which might have caused a temporary rise in intraocular pressure - for example, trauma, uveitis, treatment with topical steroids, etc; examination findings suggestive of the same antecedent problems; open and normal angles on gonioscopy; no features of other ocular abnormality which might mimic the visual field or optic disc changes of glaucoma. There are also several occult conditions which might be confused with low tension glaucoma, but which are unlikely to cause continuing deterioration in the visual field. They include ischaemic optic neuropathy and branch retinal vein or arteriole occlusion which have healed without or with only minimal retinal abnormality on ophthalmoscopy. Finally, magnetic resonance imaging or computed tomography scan are essential to exclude extrinsic compression of the optic nerve or chiasm. Headaches must not be dismissed lightly in cases of suspected low tension glaucoma as they may indicate intracranial tumour or arteritis.

Once these important exclusions have been made, there may be value in the classification of low tension glaucoma into subgroups. ${ }^{6}$ Several categories can be identified including those associated with ischaemia, high myopia, and age-related atrophy, the last often associated with peripapillary atrophy of the choroid. To these we must add what is perhaps the commonest type, the iatrogenic, following successful lowering of pressure, even by surgery, in high pressure primary open angle glaucoma. Here the decay in visual field is very slow and may take up to 40 years to extinguish the whole field of vision. ${ }^{78}$ Is this merely attenuation of a depleted nerve fibre population with advancing age or is it indicative of continuing disease activity?

Study of the subgroups will perhaps reveal helpful differential features such as the presence of abnormal vascular reactivity, ${ }^{910}$ different patterns of visual field loss, ${ }^{11-13}$ or response to treatment. When medical treatment produces marked lowering of pressure, it suggests an abnormality of pressure control analogous to primary open angle glaucoma. These cases may be treated medically, but where there is progressive deterioration in visual field in normal tension glaucoma we have increasing support from the present paper and from others ${ }^{1415}$ that drainage surgery is indicated.

There is a further lesson from ancient Greece. We must not behave like Procrustes when we consider the clinical evidence or lack of it in reaching a diagnosis of normal tension glaucoma. He would have had us distort or neglect the evidence to make patients fit a preconceived notion of the disease. (He was a robber who adjusted the size of his victims to suit his beds by stretching the short and amputating the tall.) We should be circumspect in our investigation of this disease and observe its progress carefully knowing that, for appropriate patients, surgical treatment is effective.

Tennent Institute of Ophthalmology,

JEFFREY L JAY

University of Glasgow,

Glasgow G11 6NT 
1 Kahn HA, Milton RC. Alternative definitions of open angle glaucoma. Effect on prevalence and associations in the Framingham eye study. Arch Ophthalmol 1980; 98: 2172-7.

2 Sponsel WE. Tonometry in question: can visual screening tests play a more decisive role in glaucoma diagnosis and management? Surv Ophthalmol 1988; 33 (suppl): 291-300.

3 Rosetti L, Marchetti I, Orzales N, Scorpiglione N, Torri V, Liberati A. Randomized clinical trials of medical treatment of glaucoma, are they appropriate to guide clinical practice? Arch Ophthalmol 1993; 111: 96-103.

4 Jay JL. Medical versus surgical treatment of primary open angle glaucoma. In: Davidson SI, Jay B, eds. Recent advances in ophthalmology. Edinburgh: In: Davidson SI, Jay B, eds. Recent advanc

5 Wilensky JT, Gieser DK. Low tension glaucoma. In: Weinstein GW, ed. Open angle glaucoma. Edinburgh: Churchill Livingstone, 1986: 49-65.

6 Geijssen $\mathrm{CH}$. Studies on normal pressure glaucoma. Amsterdam: Kugler, 1991: 202-9.
7 Smith RJH. The enigma of primary open angle glaucoma. Trans Ophthalmol Soc UK 1986; 105: 618-33.

8 Jay JL, Murdoch JR. The rate of visual field loss in untreated primary open angle glaucoma. Br f Ophthalmol 1993; 77: 176-8.

9 Drance SM. Glaucoma. Changing concepts. Eye 1992; 6: 337-45.

10 Jay JL. The vascular factor in low tension glaucoma: alchemists' gold? Br f Ophthalmol 1992; 72: 1 .

11 King D, Drance SM, Douglas G, Schulzer M, Wijsman K. Comparison of visual field defects in normal tension and high tension glaucoma. of visual field defects in normal tens

12 Glicklich RE, Steinmann WC, Spaeth GL. Visual field change in low tension glaucoma over a five year follow up. Ophthalmology 1989; 96: 316-20.

13 Chauhan BC, Drance SM, Douglas GR, Johnson CA. Visual field damage in normal tension and high tension glaucoma. Am $f$ Ophthalmol 1989; 108: $636-42$.

14 Bloomfield S. The results of surgery in low tension glaucoma. $A m f$ Ophthalmol 1953; 36: 1067-70.

15 de Jong, Greve EL, Hoyng PEG, Geijssen HC. Results of filtering procedure in low tension glaucoma. Int Ophthalmol 1989; 13: 131-8.

\section{The SLO, yet again}

In recent years there have been a number of papers demonstrating the versatility of the scanning laser ophthalmoscope (SLO), including its use for fluorescein angiography, ${ }^{12}$ quantification of retinal blood flow, ${ }^{3}$ static fundus controlled perimetry, ${ }^{4}$ and the measurement of optic disc variables. ${ }^{5} 6$ To add to this list we now have the imaging of fundus autofluorescence, described in this issue in the paper by von Rückmann and colleagues ( $p$ 407). They take advantage of two of the major features of the confocal SLO, the ability to image using monochromatic light and the production of an image of a relatively thin slice of tissue. Clearly, the SLO is an extremely versatile instrument; to what extent does it offer the 'ultimate' imaging experience for ophthalmologists?

The concept of using lasers to image the retina was first proposed by Webb and colleagues in $1980^{7}$; the instrument was known initially as the flying spot TV ophthalmoscope, the term 'scanning laser ophthalmoscope' being introduced the following year. The conventional fundus camera illuminates a majority of the fundus simultaneously and so requires a large entrance aperture which, in turn, limits the size of the exit aperture through which the reflected beam can pass. The result is that the illuminating beam needs to be of high intensity. In contrast the SLO uses serial imaging; a narrow beam of laser light is scanned in a raster fashion across the fundus with an image of only one small point on the fundus being made at any one time. This means that a very small input beam of laser light can be used, freeing the rest of the pupil area for the reflected light to exit through. The immediate advantage of the system is that it allows imaging to be carried out at low light levels, some 200 times lower than with the fundus camera. ${ }^{8}$

The image is intrinsically a digitised one, although commercial instruments usually record it as a video signal. The main disadvantage of the instrument is its limited spatial resolution; the optics of the eye restricting the size of the laser spot on the retina to some $15 \mu \mathrm{m}$ in diameter. Thus the SLO will lose some of the fine low contrast detail of the fundus camera. It should be noted, however, that providing the feature to be detected has sufficient contrast then it will be visible, albeit blurred, even though its actual size is much smaller than the spot size.

Apart from the convenience of being able to use low intensity light, the SLO offers a number of interesting features, several of whose potential has not yet been explored fully. The contrast of retinal features can be varied by changing the thickness of section over which reflected light is received, the amount of scattered light detected, and the wavelength of the illuminating beam. The development of the confocal version of the SLO by Webb in $1987^{\circ}$ has led to tomographic imaging and a reduction in the amount of scattered light in the image. Unfortunately, imaging at depth will always depend upon the amount of light penetrating through the overlying layers of tissue and so it is inevitable that the quality of images of the deeper fundal layers will be limited. Also the thinner the section is, the lower will be the amount of light available and, hence, the poorer the quality of the image. There are two ways of addressing this problem; to increase either the strength of the illuminating beam or the exposure time. While the difference between the exposure from the SLO and fundus camera might suggest that the power of the irradiating laser, some $50 \mu \mathrm{W}$, may be safely increased there is, in fact, little information on the effect on retinal tissue of exposure to monochromatic laser light at this level. This is an area in which more work on safe working exposure levels is required.

The solution proposed in von Rückmann et al's paper was to increase the effective exposure time, by summing multiple images. However, this introduces the problem of correcting for eye movement. Unfortunately, the registration of images requires not simply corrections for shift but also image warping, and manual alignment of the images is often not sufficient for this. Much effort has been expended upon algorithms for movement correction of retinal images, ${ }^{10}$ these are sophisticated but, as a result, often very time consuming even on modern workstations. This may prove to be the factor that limits the value of this technique.

A further problem is that there is little information as to the size of tomographic sections produced by the SLO. Although some workers have produced results measuring slice thickness, ${ }^{11-13}$ the manufacturers of commercial instruments often only identify the confocal aperture by a number and do not provide information about slice thickness. In practice, the slice thickness needed to produce an image of reasonable quality appears to be about $2500 \mu \mathrm{m},{ }^{13}$ thinner slices being too noisy. This must surely raise questions as to the value of the instrument for measuring retinal topography. While there have been published results showing the technique to be reproducible, ${ }^{14}$ measurements made on humans have had certain inconsistencies in the values produced ${ }^{6}$ which have not been convincingly explained.

Perhaps the most interesting feature of the instrument is 